\title{
Prevalencia de hormona antimülleriana en mujeres latinas atendidas en un centro de fertilidad en México
}

\author{
Héctor S. Godoy-Morales ${ }^{1,2,3 *}$, Germán G. Palacios-López ${ }^{3}$, Miguel Loyo-Guiot ${ }^{3}$, Griselda C. Reyes-Torres ${ }^{3}$, \\ Pablo J. Cervantes-Mondragón ${ }^{3}$, Daniel Vieyra-Cortés ${ }^{3}$ e Hilda Sánchez-Hernández ${ }^{3}$ \\ ${ }^{1}$ Dirección, ART Reproducción; ${ }^{2} J e f a t u r a$, Clínica de Fertilidad, Hospital Ángeles Pedregal; ${ }^{3}$ Curso de Biología de la Reproducción Humana, Hospital \\ Ángeles del Pedregal. Ciudad de México, México
}

\section{Resumen}

Antecedentes: La hormona antimülleriana (HAM) ha sido utilizada como marcador de reserva ovárica, lo cual ayuda a predecir el éxito. Se desconoce si valores de otros países son apicables a México. Objetivo: Determinar los valores de HAM en nuestra población para graficarlas acorde a grupos de edad y evaluar su comportamiento. Metodología: Estudio observacional, descriptivo, retrospectivo y transversal de pacientes del 2008 al 2018, con reporte de nivel de HAM excluyendo a pacientes con enfermedades que alteran su nivel, analizando por grupos de edad en un laboratorio. Resultados: 450 pacientes de 36.93 años, el rango del valor de la HAM fue desde $0.003 \mathrm{ng} / \mathrm{ml}$ hasta $9.4 \mathrm{ng} / \mathrm{ml}$, con una media de 1.5 , con una disminución anual a partir de los 40 años (0.1-0.3). Correlación de Spearman de -436 $(p<0.000)$ con una asociación leve entre la edad y el nivel de HAM, pero sí lineal $(p<0.000)$. Conclusiones: La HAM es útil y sirve para dar recomendaciones personalizadas, así como base de estudios epidemiológicos en pro de una medicina individualizada.

Palabras clave: Prevalencia. Edad. Hormona antimülleriana.

\section{Antimüllerian hormone prevalence in Latin women treated at a fertility center in Mexico}

\section{Abstract}

Background: Antimüllerian hormone $(\mathrm{AMH})$ has been used as a marker of ovarian reserve, which helps predict success. It is unknown if values from other countries are applicable to Mexico. Objective: To determine the AMH values in our population to graph them according to age groups and evaluate their behavior. Methodology: Observational, descriptive, retrospective, and cross-sectional study of patients from 2008 to 2018, with a AMH level report excluding patients with diseases that alter their level, analyzing by age groups in a laboratory. Results: 450 patients of 36.93 years old, the range of the AMH value was from $0.003 \mathrm{ng} / \mathrm{ml}$ to $9.4 \mathrm{ng} / \mathrm{ml}$, with a mean of 1.5, with an annual decrease from 40 years of age (0.1-0.3). Spearman's correlation of $-436(p<0.000)$ with a slight association between age and AMH level, but it was linear $(p<0.000)$. Conclusions: $A M H$ is useful and serves to give personalized recommendations, as well as basis of epidemiological studies in favor of an individualized medicine.

Key words: Prevalence. Age. Antimüllerian hormone.

\section{Correspondencia:}

*Héctor S. Godoy-Morales

E-mail: artreproduccion@gmail.com licencia CC BY-NC-ND (http://creativecommons.org/licenses/by-nc-nd/4.0/).
Disponible en internet: 28-12-2021 Perinatol Reprod Hum. 2021;35(1):10-16 www.perinatologia.mx 


\section{Antecedentes}

Para predecir el éxito de las técnicas de alta complejidad y como factores pronósticos, se han usado los marcadores de la reserva ovárica, los cuales varían considerablemente entre cada mujer, lo cual hace difícil a la vez predecir la vida productiva restante a una edad dada ${ }^{1}$, esto ha sido de especial interés sobre todo en aquellas mujeres que han retrasado sus deseos de concebir a cambio de su desarrollo profesional ${ }^{2}$. La medición directa y $100 \%$ fiable en nuestros días aún es imposible, sin embargo, la medición de folículos antrales representa cuantitativamente el aspecto del envejecimiento ovárico ${ }^{3}$.

A mayor edad, menor respuesta ovárica, independientemente de las dosis de medicamentos usados. Esto ha sido demostrado en numerosas ocasiones y se ha visto relacionado con otros factores como la obesidad o alteraciones hormonales diversas ${ }^{4}$. Existen diversos marcadores de la reserva ovárica y uno que ha tomado interés es el uso de la hormona antimülleriana (HAM), la cual es un miembro de la familia del factor transformante $\beta$, que es producido en las células de la granulosa ${ }^{5}$, y la cual refleja la disminución de los folículos primordiales mientras avanza la edad ${ }^{6}$. Tener en consideración estos aspectos y su identificación antes de iniciar un programa de reproducción asistida de alta complejidad ha ayudado tanto a la selección del protocolo adecuado como a la asesoría pertinente, por lo que han surgido con el tiempo esfuerzos por encontrar la prueba adecuada o la más certera para la evaluación correcta de la reserva ovárica ${ }^{5}$.

Los niveles más altos de HAM se han observado en folículos de hasta $6 \mathrm{~mm}$ en la pubertad y van disminuyendo conforme avanza la edad y es indetectable en la menopausia, aunque con la limitante de que es variable en cada ciclo, pero su utilidad radica en que puede servir como marcador de la reserva ovárica y a diferencia del recuento de folículos antrales es menos dependiente del operador ${ }^{7-10}$.

Una mujer con un nivel bajo de HAM tiene una baja reserva ovárica $\mathrm{y}$, por ende, está más cercana a la menopausia, comparada con otra con niveles más altos de la misma edad, aunque ha habido estudios que no apoyan este argumento, toda vez que la gran mayoría de las veces las mediciones son únicas ${ }^{11}$, se eleva en pacientes con síndrome de ovario poliquístico, lo cual es de por sí una causa de infertilidad ${ }^{12}$, debido al alto número de folículos antrales e inclusive se relaciona con el índice de masa corporal ${ }^{13}$.
Existe un estudio donde se observó en mujeres de EE.UU. los valores que llegan a tener según el grupo de edad que ha servido como guía para orientar a las pacientes del nivel aproximado que tienen de reserva ovárica a una edad dada, lo cual se ha correlacionado con la respuesta en los ciclos de reproducción asisti$\mathrm{da}^{14}$. Sin embargo, estas variaciones han hecho que el uso de la HAM sea controvertido, sobre todo en lugares como en nuestra población, donde su uso ha sido poco extendido debido a su variabilidad y su costo, lo que hizo que años atrás no fuera rentable, pero hoy en día y tras las evidencias acumuladas en los diversos estudios es una herramienta cada vez más usada, como en nuestro centro, donde es parte del protocolo inicial a las pacientes que acuden por infertilidad, sobre todo en aquellas que van a ser sometidas a tratamientos reproductivos de alta complejidad.

En nuestro país no tenemos una curva de prevalencia según la edad, por lo que el objetivo del presente estudio es valorar los resultados de todas las pacientes a que se les haya tomado muestra de dicha hormona en un esfuerzo por correlacionar los niveles según la edad de las pacientes y su comportamiento a lo largo del tiempo.

\section{Material y métodos}

Estudio de tipo observacional, descriptivo, retrospectivo y transversal en el cual se obtuvieron los registros de pacientes atendidas en la clínica de fertilidad del Hospital Ángeles Pedregal de la Ciudad de México desde el 2008 y hasta el 2018, utilizando los expedientes del archivo clínico. Se incluyeron a pacientes con diagnostico de infertilidad de cualquier tipo y por cualquier factor, sin límite de edad y que tuvieran expedientes completos. Se excluyeron los casos con información insuficiente o que no tuvieran mediciones de HAM. Debido a que el objetivo era hacer una curva de dicha hormona conforme a la evolución de la edad, consideramos también excluir a pacientes con enfermedades que alteran el nivel de esta, para disminuir sesgos (síndrome de ovarios poliquísticos, endometriosis con endometrioma, cirugías previas de ovario y pacientes ooforectomizadas). Las fuentes de información del estudio fueron la historia clínica obtenida del expediente y una cédula de recolección de datos diseñada ad hoc.

Para cada caso se recolectaron las variables edad, estudios hormonales basales tomados en el día 2 del ciclo menstrual (incluyendo HAM y hormona foliculoestimulante $[\mathrm{FSH}]$ ), y se describieron los antecedentes de 
Tabla 1. Características de las pacientes con padecimientos que modifican los niveles de hormona antimülleriana

\begin{tabular}{|l|c|c|c|c|}
\hline Patología & Rango de edad (años) & Nivel mínimo & Nivel máximo & Media \\
\hline Síndrome de ovarios poliquísticos $(\mathrm{n}=40)$ & $19-46$ & 0.32 & 12 & 1.86 \\
\hline Cirugías previas de ovarios $(\mathrm{n}=26)$ & $26-40$ & 0.023 & 6.74 & 1.80 \\
\hline solo ovario $(\mathrm{n}=21)$ & $19-44$ & 0.032 & 5.3 & 1.84 \\
\hline Endometriosis con endometrioma $(\mathrm{n}=12)$ & $32-42$ & 0.16 & 5.94 & 1.57 \\
\hline
\end{tabular}

importancia como endometriosis, antecedentes de infertilidad y alteraciones anatómicas, así como el tipo de infertilidad, factores causales y el tiempo de evolución de esta. En el caso de la toma de los estudios séricos, las mediciones de HAM se determinaron en un solo laboratorio de referencia (LabCorp), avalado y aprobado por CENARE S.A. de C.V.

El muestreo fue de tipo no probabilístico convencional y el método de investigación utilizado fue la observación y recolección de datos por medio de un registro, utilizando el instrumento de la cédula de recolección de información. Para el análisis estadístico de los resultados se emplearon programas de paquetes de datos (Microsoft Excel $^{\odot}$ y IBM SPSS Statistics ${ }^{\odot}$ versión 20 ) mediante estadística descriptiva utilizando medidas de tendencia central y de dispersión para las variables cuantitativas y con frecuencias y proporciones para las variables cualitativas. Así mismo, para el cálculo de correlación entre las variables edad y HAM, así como edad y FSH, se utilizó primero la prueba para determinar la normalidad de los datos (Kolmogorov-Smirnov), pero debido a carecer de homocedasticidad, se utilizó la correlación de Spearman para variables cuantitativas de distribución no gaussiana.

Este estudio fue autorizado por el Comité de Investigación del Hospital Ángeles Pedregal y se encuentra estrictamente apegado a los lineamientos vigentes de la Ley General de Salud en el Capítulo I de los Aspectos Éticos de la Investigación en Seres Humanos en su artículo 17.

\section{Resultados}

Se analizaron un total de 720 expedientes de pacientes atendidas por infertilidad en este centro por el autor del artículo, en el tiempo establecido, descartándose 160 por no tener registro de HAM, así como 11 por no contar con el registro de la edad. Debido a que el objetivo era hacer una curva de dicha hormona conforme a la evolución de la edad se hizo un análisis breve y por separado de las pacientes con enfermedades que alteran el nivel de la hormona (síndrome de ovarios poliquísticos, endometriosis con endometrioma, cirugías previas de ovario y ooforectomizadas), las características principales de estas pacientes se resumen en la tabla 1.

Posterior a la exclusión de estos casos, se obtuvo una muestra final de 450 pacientes, donde el rango de edad fue de 18 a 48 años, con una media de 36.93 años y una desviación estándar (DE) de 5.2. El motivo de consulta más frecuente fue por infertilidad primaria $(58.8 \%)$, con un tiempo de infertilidad promedio de 45 meses, DE 40 meses, con un mínimo de 12 y máximo de 210 meses; en cuanto a factores de infertilidad, se distribuyeron conforme a lo observado en la tabla 2.

El rango del valor de la HAM fue desde 0.003 hasta $9.4 \mathrm{ng} / \mathrm{ml}$, con una media de $1.5 \mathrm{ng} / \mathrm{ml}$ y una DE de 1.82. Se realizaron segmentaciones por grupos de edad, debido al bajo número de casos debajo de los 30 años (es decir, se incluyeron a todos los casos de los 18 a los 30 años), posteriormente se describió por cada edad los valores de antimülleriana (Tabla 3). Se logró observar una disminución anual a partir de los 40 años, de entre 0.1 y $0.3 \mathrm{ng} / \mathrm{ml}$ (Fig. 1). Al realizar las pruebas estadísticas para determinar si existía una relación entre la edad y el nivel de HAM, se realizaron gráficos de dispersión para valorar el panorama general de los datos, encontrando una amplia y no lineal, por lo que se procedió a realizar la prueba de Kolmogorov-Smirnov, encontrándolos con distribución no normal $(p<0.000)$. En ese caso se procedió a utilizar la correlación de Spearman para variables cuantitativas continuas de distribución libre, encontrando un coeficiente de correlación de -436 , con una significancia de 0.000 , por lo que se determinó que existía una asociación leve entre la edad y el nivel de la HAM conforme avanzaba esta última, pero sí lineal $(p<0.000)$. 
Tabla 2. Motivos de consulta en la clínica de fertilidad

\begin{tabular}{|l|c|}
\hline Factor de infertilidad & Porcentaje \\
\hline Edad (> 40 años) & $35.8 \%$ \\
\hline Factor uterino & $9.3 \%$ \\
\hline Factor cervical & $1 \%$ \\
\hline Factor tubárico & $11.9 \%$ \\
\hline $\begin{array}{l}\text { Baja reserva (< } 4 \text { folículos antrales) } \\
\text { Baja respondedora (<3 ovocitos capturados en } \\
\text { un ciclo de FIV a dosis convencionales previo a } \\
\text { pacientes sin características de bajas } \\
\text { respondedoras) }\end{array}$ & $14.5 \%$ \\
\hline $\begin{array}{l}\text { Factor ovárico } \\
\text { Factor masculino }\end{array}$ & $0.5 \%$ \\
\hline
\end{tabular}

FIV: fecundación in vitro.

En el caso de la FSH, otro valor hormonal que en concentraciones elevadas (> $20 \mathrm{UI} / \mathrm{l}$ ) simboliza la falla ovárica (Fig. 2), se realizó el mismo análisis, determinando que tuvo un coeficiente de correlación de Spearman de 0.142 , con una asociación de igual manera leve, pero en este caso sin seguir una distribución lineal $(p=0.23)$.

\section{Discusión}

Definir parámetros hormonales en poblaciones específicas refleja la evolución a lo largo del tiempo del cuerpo humano y permite realizar una medicina más personalizada, asesorar a las pacientes con tratamientos específicos y dar un panorama general tanto de pronóstico como lo que se espera en respuesta al tratamiento, por ejemplo, en reproducción asistida, como hemos visto con el uso cada vez más extenso de la HAM, la cual ahora se acepta como un marcador clínico importante de la reserva ovárica y se mide cada vez más como una evaluación inicial en clínicas de infertilidad. Sin embargo, no se han informado valores de referencia específicos de la edad para pacientes mexicanas derivados de un gran conjunto de datos ${ }^{15}$.

Este marcador ha sido utilizado junto con otros parámetros para definir los criterios de baja respuesta y ha ayudado a hacer un consenso sobre quiénes son las pacientes que tendrán una menor reacción a los tratamientos de reproducción asistida ${ }^{16}$. Derivado de ello surgió el interés de realizar estudios en las poblaciones donde se han encontrado variaciones conforme avanza la edad. En nuestra población no existe una curva del valor hormonal que refleje el comportamiento

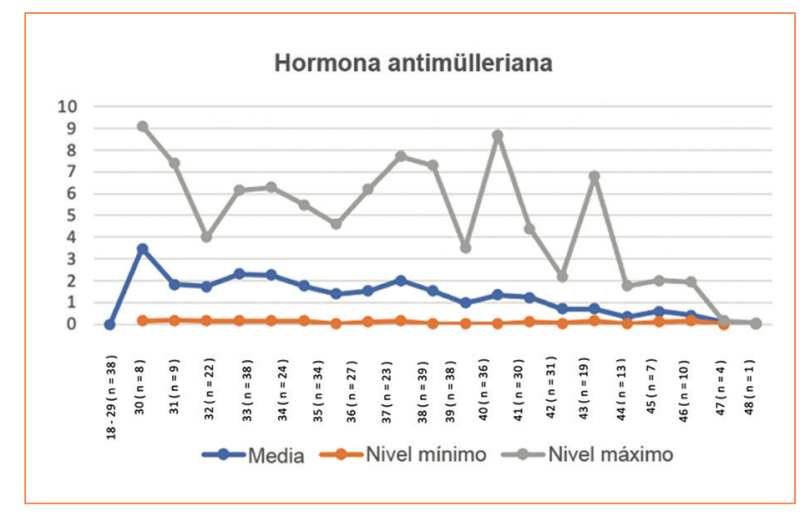

Figura 1. Distribución de los valores medio, mínimo y máximo de la hormona antimülleriana $(\mathrm{ng} / \mathrm{ml})$ por grupos de edad.

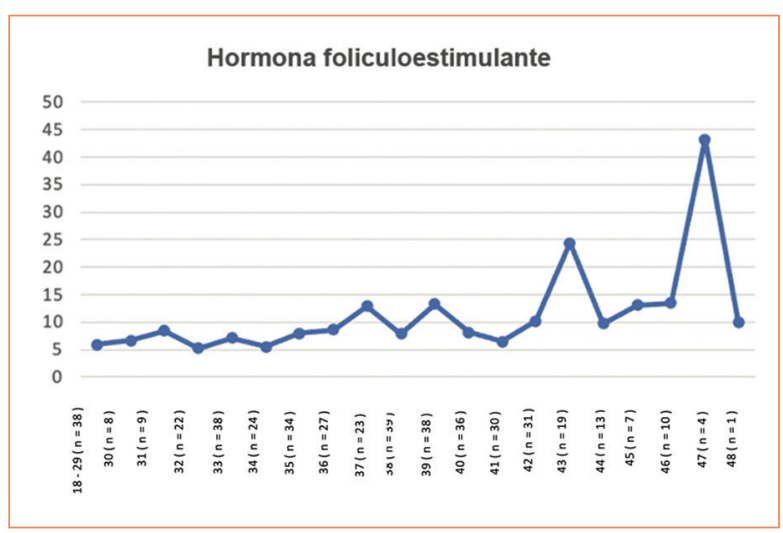

Figura 2. Nivel medio de la hormona foliculoestimulante (IU/I) por grupo de edad.

específico de nuestra población, toda vez que en nuestro país no existen estudios extensos de la HAM, su prevalencia y su distribución por edades específicas.

Existe un estudio en EE.UU. donde se obtuvieron los registros de 17,120 pacientes atendidas en diversas clínicas de fertilidad de ese país, donde fue evidente que al pasar del tiempo la disminución de la HAM fue de 0.1 a $0.2 \mathrm{ng} / \mathrm{ml}$ por año a partir de los 38 años, este estudio tuvo la virtud de tener las mediciones de un solo laboratorio ${ }^{17}$, a diferencia de estudios en Europa donde se analizaron menos pacientes y con la variación de que tuvieron muestras tomadas en laboratorios diferentes ${ }^{18,19}$. En nuestro estudio pudimos observar un comportamiento similar determinando que a partir de los 40 años las disminuciones son marcadas, pasando a ser prácticamente indetectables para los 45 años y más, teniendo la virtud que al igual que en el estudio 
Tabla 3. Distribución por grupo de edad de los valores de la HAM (ng/ml)

\begin{tabular}{|c|c|c|c|c|c|c|}
\hline Rango de edad años (n) & Nivel mínimo & Nivel máximo & Media HAM & DE HAM & Media FSH & DE FSH \\
\hline $18-29(n=38)$ & 0.16 & 9.10 & 3.47 & 2.93 & 5.92 & 30 \\
\hline $30(n=8)$ & 0.180 & 7.4 & 1.82 & 2.37 & 6.62 & 4.6 \\
\hline $31(n=9)$ & 0.160 & 4 & 1.74 & 1.39 & 8.4 & 2.7 \\
\hline $32(n=22)$ & 0.160 & 6.16 & 2.31 & 1.67 & 5.2 & 2.3 \\
\hline $33(n=38)$ & 0.160 & 6.3 & 2.25 & 1.75 & 7.1 & 2.9 \\
\hline $34(n=24)$ & 0.160 & 5.5 & 1.76 & 1.53 & 5.5 & 1.75 \\
\hline $35(n=34)$ & 0.023 & 4.6 & 1.38 & 1.25 & 7.97 & 4.3 \\
\hline $36(n=27)$ & 0.100 & 6.2 & 1.52 & 1.67 & 8.6 & 3.8 \\
\hline $37(n=23)$ & 0.160 & 7.7 & 2.00 & 2.23 & 12.9 & 27.9 \\
\hline $38(n=39)$ & 0.023 & 7.3 & 1.52 & 1.7 & 7.9 & 6.0 \\
\hline $39(n=38)$ & 0.017 & 3.5 & 0.97 & 0.94 & 13.3 & 23 \\
\hline $40(n=36)$ & 0.023 & 8.7 & 1.34 & 1.97 & 8.1 & 9.1 \\
\hline $41(n=30)$ & 0.100 & 4.4 & 1.23 & 1.27 & 6.5 & 3.7 \\
\hline $42(n=31)$ & 0.030 & 2.2 & 0.70 & .060 & 10.2 & 10.7 \\
\hline $43(n=19)$ & 0.160 & 6.8 & 0.70 & 1.51 & 24.4 & 48.3 \\
\hline $44(n=13)$ & 0.015 & 1.75 & 0.34 & 0.45 & 9.7 & 12.9 \\
\hline $45(n=7)$ & 0.100 & 2.0 & 0.60 & 0.74 & 13.04 & 10.04 \\
\hline $46(n=10)$ & 0.160 & 1.94 & 0.40 & 0.54 & 13.49 & 12.7 \\
\hline $47(n=4)$ & 0.003 & 0.160 & 0.12 & 0.07 & 43.2 & 19.9 \\
\hline $48(n=1)$ & 0.03 & 0.03 & 0.03 & & 10 & \\
\hline
\end{tabular}

DE: desviación estándar; HAM: hormona antimülleriana; FSH: hormona foliculoestimulante.

realizado en EE.UU., las muestras de laboratorio de todas nuestras pacientes fueron tomadas de un mismo laboratorio de referencia.

Debido a que se han observado alteraciones marcadas bien establecidas de la HAM en patologías y situaciones específicas como síndrome de ovarios poliquísticos ${ }^{20}$, cirugías previas de ovarios ${ }^{21}$, presencia de un solo ovario ${ }^{22}$ y endometriosis (con mayor impacto en pacientes con endometrioma) $)^{23,24}$, en nuestro estudio se excluyeron las pacientes que contaran con este tipo de patologías, esto le da un valor adicional, toda vez que en los estudios previamente mencionados no estratificaron a las pacientes con estos antecedentes y se las incluyó en el análisis general, aunque si bien esto no hizo que variara mucho con nuestro datos, al menos en las medias observadas, sí lo hizo al momento de observar las variaciones en los rangos máximos y mínimos, cosa que es importante hacer notar y sobre todo mencionar a las pacientes a la hora de brindarles asesoría y entregarles el reporte del estudio.

A pesar de las fortalezas antes mencionadas, una limitación de nuestro estudio fue el tamaño de muestra, que es mucho menor que el más extenso realizado en EE.UU., sin embargo, es de notar que nuestro centro de fertilidad fue el primero en describir el comportamiento de la HAM en una población donde se obtuvieron los registros de 39 pacientes y se comparó con los niveles de hormona foliculoestimulante ${ }^{24}$. Desde entonces se observaba que había variaciones conforme a la edad; hoy podemos aumentar la evidencia que tenemos de que la utilización de la hormona es realmente útil y que si una mujer joven tiene niveles de los de una edad mayor, será una paciente que evidentemente puede llegar a responder inadecuadamente a nuestros tratamientos. Además, todos los datos obtenidos juegan en pro de una medicina más personalizada y efectiva que evite el dispendio de recursos y 
ajustada a nuestra población. Por último, mencionar que la distribución anormal de los datos no nos permitió realizar un cálculo de un coeficiente de determinación que nos podría haber dado datos más concretos de este comportamiento, pero podemos al menos afirmar que en nuestra población la disminución de la HAM conforme pasa el tiempo tiene una relación lineal respecto a la edad, aunque con una asociación leve.

\section{Conclusiones}

Determinar los valores de HAM sigue siendo útil en el estudio de las pacientes que son atendidas en las clínicas de fertilidad. Realizar estudios en poblaciones específicas servirá como base para estudios epidemiológicos, realizar asesorías personalizadas a las pacientes brindará datos específicos en nuestras pacientes que comparten características distintas a las de otros países en diversos ámbitos ya conocidos tanto genéticos como ambientales. La disminución de la HAM conforme el paso de la edad brinda evidencia para un grupo de mujeres que busca cada vez más la autorrealización profesional y postergan la maternidad, las cuales deben ser asesoradas en tiempo y forma sobre su futuro reproductivo con datos reales y de acuerdo con mujeres que comparten estas mismas características, pues son parte de una misma población, para que tomen decisiones oportunas en pro de su futuro reproductivo. Juntar más datos de pacientes, realizar estudios más extensos y según cada situación particular (las diferentes enfermedades ya mencionadas) debe ser la meta para la medicina del futuro, que será individual y personalizada, ya que a pesar de todo la amplia variabilidad en los niveles obtenidos debe ser, a pesar de todo, algo que aún debe llamarnos la atención y eliminar las dudas al respecto, solo puede hacerse mediante estudios más amplios.

\section{Agradecimientos}

Agradezco a mi profesor titular, quien nos ha brindado su apoyo incondicional, así como a todos los integrantes de ART Reproducción por la colaboración para crear nuestro artículo.

\section{Financiamiento}

La presente investigación no ha recibido ninguna beca específica de agencias de los sectores públicos, comercial o con ánimo de lucro.

\section{Conflicto de intereses}

Los autores declaran no tener conflicto de intereses.

\section{Responsabilidades éticas}

Protección de personas y animales. Los autores declaran que para esta investigación no se han realizado experimentos en seres humanos ni en animales.

Confidencialidad de los datos. Los autores declaran que han seguido los protocolos de su centro de trabajo sobre la publicación de datos de pacientes.

Derecho a la privacidad y consentimiento informado. Los autores han obtenido el consentimiento informado de los pacientes y/o sujetos referidos en el artículo. Este documento obra en poder del autor de correspondencia.

\section{Bibliografía}

1. Te Velde ER, Pearson PL. The variability of female reproductive aging. Hum Reprod Update. 2002;8:141-54.

2. Leridon $H$. Demographic effects of the introduction of steroid contraception in developed countries. Hum Reprod Update. 2006;12:603-16.

3. Broekmans FJ, Kwee J, Hendriks DJ, Mol BW, Lambalk CB. A systematic review of tests predicting ovarian reserve and IVF outcome. Hum Reprod Update. 2006;12:685-718.

4. De Koning $\mathrm{CH}$, Benjamins T, Harms $\mathrm{P}$, Homburg R, van Montfrans JM, Gromoll J, et al. The distribution of $\mathrm{FSH}$ receptor isoforms is related to basal FSH levels in subfertile women with normal menstrual cycles. Hum Reprod. 2006;21:443-6.

5. Hendriks DJ, Mol BW, Bancsi LF, Te Velde ER, Broekmans FJ. Antral follicle count in the prediction of poor ovarian response and pregnancy after in vitro fertilization: a meta-analysis and comparison with basal follicle-stimulating hormone level. Fertil Steril. 2005;83:291-301.

6. Lee MM, Donahoe PK, Hasegawa T, Silverman B, Crist GB, Best S, et al. Mullerian inhibiting substance in humans: normal levels from infancy to adulthood. J Clin Endocrinol Metab. 1996;81:571-6.

7. Hansen KR, Hodnett GM, Knowlton N, Craig LB. Correlation of ovarian reserve tests with histologi- cally determined primordial follicle number. Fertil Steril. 2010:10:60-84

8. Weenen C, Laven JS, von Bergh AR, Cranfield M, Groome NP, Visser JA, et al. Anti-mullerian hormone expression pattern in the human ovary: potential implications for initial and cyclic follicle recruitment. Mol Hum Reprod. 2004;10:77-83.

9. Gruijters MJ, Visser JA, Durlinger AL, Themmen AP. Anti-mullerian hormone and its role in ovarian function. Mol Cell Endocrinol. 2003;211: 85-90.

10. Hehenkamp WJ, Looman CW, Themmen AP, de Jong FH, Te Velde ER, Broekmans FJ. Anti-mullerian hormone levels in the spontaneous menstrual cycle do not show substantial fluctuation. J Clin Endocrinol Metab. 2006:91:4057-63.

11. Eifer DB, MacLaughlin DT, Christian BP, Feng B, Shelden RM. Early follicular serum mullerian-inhibiting substance levels are associated with ovarian response during assisted reproductive technology cycles. Fertil Steril. 2002;77:468-71

12. Doroftei $B$, Mambet $C$, Zlei M. It's never over until it's over: how can age and ovarian reserve be mathematically bound through the measurement of serum HAM-A study of 5069 Romanian women. PLoS One. 2015;10: e0125216.

13. Homburg R, Ray A, Bhide P, Gudi A, Shah A, Timms P, et al. The relationship of serum anti-Mullerian hormone with polycystic ovarian morphology and polycystic ovary syn drome: a prospective cohort study. Hum Reprod. 2013;28(4):1077-83.

14. Kriseman M, Mills C, Kovanci E, Sangi-Haghpeykar H, Gibbons W. Antimullerian hormone levels are inversely associated with body mass index (BMI) in women with polycystic ovary syndrome. J Assist Reprod Genet. 2015;32:1313-6 
15. van Helden J. Age-independent anti-Müllerian hormone (HAM) standard deviation scores to estimate ovarian function. Eur J Obstet Gynecol Reprod Biol. 2017;213:64-70.

16. Ferraretti AP, La Marca A, Fauser BC, Tarlatzis B, Nargund G, Gianaroli L; ESHRE consensus on the definition of 'poor response' to ovarian stimulation for in vitro fertilization: the Bologna criteria. Human Reproduction. 2011;26:16-24

17. Seifer DB, Baker VL, Leader B. Age-specific serum anti-Mullerian hormone values for 17,120 women presenting to fertility centers within the United States. Fertil Steril. 2011;95(2):747-50.

18. Nelson SM, Messow MC, Wallace AM, Fleming R, McConnachie A Nomogram for the decline in serum antimullerian hormone: a population study of 9,601 infertility patients. Fertil Steril. 2011;95(2):736-41.e1-3.

19. La Marca A, Sighinolfi G, Giulini S, Traglia M, Argento C, Sala C, et al. Normal serum concentrations of anti-Mullerian hormone in women with regular menses cycles. Reprod Biomed Online. 2010;4:463-9.

20. Qi X, Pang Y, Qiao J. The role of anti-Müllerian hormone in the pathogenesis and pathophysiological characteristics of polycystic ovary syndrome. Eur J Obstet Gynecol Reprod Biol. 2016;199: $82-7$
20. Chung J, Law T, Chung C, Mak J, Sahota DS, Li TC. Impact of haemostatic sealant versus electrocoagulation on ovarian reserve after laparoscopic ovarian cystectomy of ovarian endometriomas: a randomised controlled trial. BJOG. 2019:126(10):1267-75.

21. Rustamov O, Krishnan M, Roberts SA, Fitzgerald CT. Impact of hemostatic sealant versus electrocoagulation on ovarian reserve after laparoscopic ovarian cystectomy of ovarian endometriomas: a randomised controlled trial. Effect of salpingectomy, ovarian cystectomy and unilateral salpingo-oopherectomy on ovarian reserve. Gynecol Surg. 2016;13: 173-8.

22. Pacchiarotti A Evaluation of serum anti-Mullerian hormone levels to assess the ovarian reserve in women with severe endometriosis. Eur $J$ Obstet Gynecol Reprod Biol. 2014;172:62-4.

23. Younis JS. Impact of unilateral versus bilateral ovarian endometriotic cystectomy on ovarian reserve: a systematic review and meta-analysis. Hum Reprod Update. 2019;25(3):375-91.

24. Godoy Morales HS, Ulloa Aguirre A, Falcón Martínez JC, Marsa Martínez EE, Rivas López R, García Lascuraín LC. Hormona antimulleriana como marcador de respuesta ovárica en fertilización in vitro. Ginecol Obstet Mex. 2012;80(1):1-7. 